\title{
COMPACT LIE GROUP ACTIONS ON ASPHERICAL $A_{k}(\pi)-M A N I F O L D S^{1}$
}

\author{
DINGYI TANG
}

\begin{abstract}
Let $M$ be an aspherical $A_{k}(\pi)$-manifold and $\pi^{\prime}$ torsion-free, where $\pi^{\prime}$ is some quotient group of $\pi$. We prove that (1) Suppose the Euler characteristic $\chi(M) \neq 0$ and $G$ is compact Lie group acting effectively on $M$, then $G$ is finite group (2) The semisimple degree of symmetry of $M N_{T}^{s} \leq(n-k)(n-k+1) / 2$. We also unity many well-known results with simpler proofs.
\end{abstract}

\section{Introduction and Preliminaries}

A CW complex (resp. manifold) is called an aspherical complex (resp. aspherical manifold) if its covering space is contractible. An extremely important class of aspherical complexes is the class of the Eilenberg-MacLane spaces $K(\pi, 1)$. A well-known theorem of Cartan-Hadamard showed that if a cnnected complete Riemannian $n$-manifold $M$ has sectional curvature $\mathbb{K}_{M} \leq 0$, then the universal covering space of $M$ is diffeomorphic to Euclidean space $\mathbb{R}^{n}$. Hence it is a $K(\pi, 1)$-space with $\pi=\pi_{1}(M)$.

We will always assume that $M$ is a compact connected oriented $n$-manifold unless otherwise stated. Whether or not there exists a non-trivial continuous map $f: M^{n} \rightarrow K(\pi, 1)$ for some group $\pi$ is an extremely important problem. For instance, Gromov and Lawson have verified that if $M$ is a spin manifold and

Received February 18, 1992; revised March 8, 1993.

1 The resuts presented here are of a doctoral dissertation written under Professor H. T. $\mathrm{Ku}$. The principal results of this dissertation will be published elsewhere. 
there exists a map $f: M \rightarrow T^{n}$ of positive degree, then $M$ does not admit a Riemannian metric with positive scalar curvature [5].

By an aspherical $A_{k}(\pi)$-manifold, we mean a compact connected $n$-manifold $M$ together with a continuous map $f: M \rightarrow K(\pi, 1)$ such that

$$
f_{*}: H_{k}(M ; Q) \rightarrow H_{k}(K(\pi, 1) ; Q)
$$

is non-trivial. Equivalently, we also can define an aspherical $A_{k}(\pi)$-manifold by using cohomology, that is, the homomorphism

$$
f^{*}: H^{k}(K(\pi, 1) ; Q) \rightarrow H^{k}(M ; Q)
$$

is non-trivial. Throughout this paper, we shall use these notations and the Alexander-Spanier cohomology with compact supports.

The group actions on the aspherical $A_{k}(\pi)$-manifold $M$ is a very important problem in topology and geometry. In 1943 [12], Montgomery and Samelson proved that if a compact Lie group acts transitively and effectively on the $n$-torus $T^{n}=M=K(Z \oplus \cdots \oplus Z, 1)$ ( $n$ copies of $\left.Z\right)$, (where $f$ is the identity), then $G \cong T^{n}$, and $G$ acts freely on $T^{n}$. Since then this problem has been investigated by many mathematicians such as Donnely-Schultz [4], Conner-Montgomery [3], H. T. Ku and M. C. Ku [8], [9], Scheon-Yau [13], etc. In this paper, we shall prove some new results concerning compact Lie group actions on aspherical $A_{k}(\pi)$ manifolds. As a by product, we are able to give new simple proofs for some well-known results.

Now, let $G$ be a compact Lie group acting on $M$. Define the map $i$ : $G \rightarrow M$ by $i(x)=g(x)$ for every $g \in G$, where $x$ is any fixed base point. Let $p: M \rightarrow M / G$ be the orbit map, and $r$ the dimension of any principal orbit. If $f: M \rightarrow K(\pi, 1)$ is continuous, it is well known that $f_{*} i_{*} \pi_{1}(G) \subset$ center of $\pi$. Hence we can define the quotient group $\pi^{\prime}=\pi / f_{*} i_{*} \pi_{1}(G)$. Let $\alpha: \pi \rightarrow \pi^{\prime}$ be the quotient map.

Lemma 1.1. Let $f: M \rightarrow K(\pi, 1)$ be a continuous map. Suppose that $\pi^{\prime}$ is torsion free. Then there exists a continuous map

$$
h: M / G \rightarrow K\left(\pi^{\prime}, 1\right),
$$


such that $p h \simeq \alpha f$ (that is, ph is homotopic to $\alpha f$ ), where $\alpha: K(\pi, 1) \rightarrow K\left(\pi^{\prime}, 1\right)$ is induced by $\alpha: \pi \rightarrow \pi^{\prime}$. In particular

$$
\alpha_{*} f_{*} H_{i}(M ; L)=0, \quad \text { for } \quad i>n-r
$$

where $L$ is any integral domain.

Proof. By [4], $\pi_{1}(M / G) \cong\left[\pi(M) / i_{*} \pi(G)\right] / N$, where $N$ is generated by a set of elements of finite order. Since $\pi^{\prime}$ is torsion-free, hence $\operatorname{Ker} p_{*} \subset \operatorname{Ker}(\alpha f)_{*}$, where

$p_{*}: \pi(M) \rightarrow \pi_{1}(M / G)$. By [4], there exists a continuous map

$h: M / G \rightarrow K\left(\pi^{\prime}, 1\right)$ such that $p h \simeq \alpha f$. Thus,

$h_{*} p_{*}=\alpha_{*} f_{*}: H_{*}(M ; L) \rightarrow H_{*}\left(K^{*}\left(\pi^{\prime}, 1\right) ; L\right)$.

As $H_{i}(M / G ; L)=0$ for $i>n-r$, because $\operatorname{dim} M / G=n-r$. The result follows. Observe that the conclusion of Lemma 1.1 remains true if $\pi$ is torsion free. Then we have a map $h: M / G \rightarrow K(\pi, 1)$ such that $p h \simeq f$ and $f_{*} I_{i}(M ; L)=0$ for $i>n-r$.

Corollary 1.2. Under the hypotheses of Lemma 1.1, if $f_{*} i_{*} \pi_{1}(G)$ is finite, then

$$
f_{*} H_{i}(M ; Q)=0, \quad \text { for } \quad i>n-r
$$

In particular, this conclusion always holds if the action of $G$ has a fixed point.

Proof. We have a fibre space

$$
K\left(f_{*} i_{*} \pi_{1}(G), 1\right) \rightarrow K(\pi, 1) \stackrel{\alpha}{\rightarrow} K\left(\pi^{\prime}, 1\right) .
$$

Since $f_{*} i_{*} \pi_{1}(G)$ is a finite group, $H_{i}\left(K\left(f_{*} i_{*} \pi_{1}(G), 1\right) ; Q\right)=0$ for $i>0$. Hence by the Vietoris-Begle mapping theorem, we have isomorphisms

$$
\alpha_{*}: H_{i}(K(\pi, 1) ; Q) \simeq H_{i}\left(K\left(\pi^{\prime}, 1\right) ; Q\right), \quad \text { for } \quad i \geq 0
$$

This will imply the desired result by Lemma 1.1. 


\section{Toral Group Actions on Aspherical $A_{k}(\pi)$-manifolds}

Let $G=T^{l}$ be a toral group acting on a compact Poincaré duality space or a rational cohomology $n$-manifold $M$. Let $E_{G}$ denote the universal space of $G$ and $M_{G}=E_{G} \times_{G} M$. Let $i: M \rightarrow M_{G}$ be the inclusion and $p: M \rightarrow M / G$ be the orbit projection as above. Following [11], for $z \in H^{s}(M / G ; Q)$ and $w \in \operatorname{Im}\left\{i^{*}: H^{n-s}\left(M_{G} ; Q\right) \rightarrow H^{n-s}(M ; Q)\right\}$, the rational number

$$
p(w, x)=\left\langle w \cup p^{*}(z),[M]\right\rangle
$$

is called a characteristic number of the orbit map, where $[M]$ denotes the fundamental homology class of $M$. If $M$ is a smooth manifold, $w$ is a product of Pontrjagin classes of $M$, and the action is smooth, then $p(w, z)$ is simply the usual Pontrjagin number of the orbit map.

Lemma 2.1.(Ku-Ku [10]). Let $G=T^{l}$ act on a compact Poincaré duality $n$-space or a rational cohomology $n$-manifold $M$ with finite robit types. Suppose $p(w, z) \neq 0$ for some $w$ and $z \in H^{s}(M / G ; Q)$. Then $H^{s}(F ; Q) \neq 0$, where $F=F(G, M)$ denotes the fixed point set.

Let $\pi^{\prime}=\pi / f_{*} i_{*} \pi_{1}(G)$ as above.

Theorem 2.2. Let $M$ be an aspherical $A_{k}(\pi)$-manifold and $\pi^{\prime}$ torsion-free. Suppose that the Euler Characteristic $\chi(M)$ is non-zero, or $p(w, z) \neq 0$ for some $w$ and $z$. Then

(1) $k \leq n-r$;

(2) There is a component $F_{0}$ of the fixed point set $F=F(G ; M)$. which is an aspherical cohomology $A_{k}(\pi)$-manifold.

Proof. The fixed point set $F$ is not empty because $\chi(F)=\chi(M) \neq 0$, or by Lemma 2.1 because $p(w, z) \neq 0$. Thus, from Corollary 1.2 we have

$$
f_{*} H_{i}(M ; Q)=0, \quad \text { for } \quad i>n-r .
$$

But $f_{*}: H_{k}(M ; Q) \rightarrow H_{K}(K(\pi, 1) ; Q)$ is non-trivial because $M$ is an aspherical $A_{K}(\pi)$-manifold. Therefore, $K \leq n-r$. This proves (1). 
To prove (2), let $F=\cup_{j=1}^{s} F_{j}$, where $F_{j}$ 's are components of $F$, and let $f_{j}=f \mid F_{j}: F_{j} \rightarrow K(\pi, 1)$. By Smith theory, each component $F_{j}$ is a cohomology manifold. Suppose that

$$
F_{j}^{*}: H^{k}(K(\pi, 1) ; Q) \rightarrow H^{k}\left(F_{j} ; Q\right)
$$

is trivial for every $j=1,2, \cdots, m$. We shall proceed to get a contradiction. From Lemma 1.1, there is a map $h: M / G \rightarrow K\left(\pi^{\prime}, 1\right)$ such that $h p \simeq \alpha f$. Let $u: F \rightarrow M$ and $v: F \rightarrow M / G$ be inclusions. Then

$$
v^{*} h^{*}=(h v)^{*}=(h p u)^{*}=(\alpha f u)^{*}=\sum_{j=1}^{m} f_{j}^{*} \alpha^{*}=0
$$

in degree $k$. Since $M$ is an aspherical $A_{k}(\pi)$-manifold and $\alpha^{*}: H^{k}\left(K\left(\pi^{\prime}, 1\right) ; Q\right)$ $\rightarrow H^{k}(K(\pi, 1) ; Q)$ is an isomorphism, hence

$$
f^{*} \alpha^{*}: H^{k}\left(K\left(\pi^{\prime}, 1\right) ; Q\right) \rightarrow H^{k}(M ; Q)
$$

is non-trivial. Let $\bar{y} \in H^{k}(K(\pi, 1) ; Q)$ be such that $f^{*} \alpha^{*}(\bar{y})=y \neq 0$. Since $v^{*} h^{*}(\bar{y})=0$, from the exact cohomology sequence of the pair $(M / G, F)$, there exists an element $y^{\prime} \in H^{*}((M-F) / G ; Q)$ such that $j^{\prime *}\left(y^{\prime}\right)=h^{*}(y)$, where $j^{\prime}$ is an inclusion. We have commutative diagram:

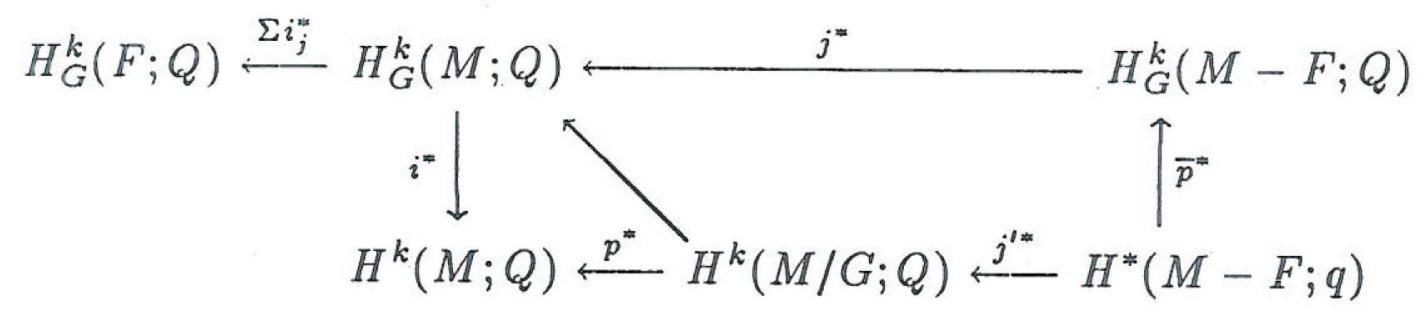

where $j$ is the inclusion, and $\bar{p}=p \mid(M-F)$. Thus we have

$$
\sum_{j=1}^{m} i_{j}^{*}\left(j^{*} \bar{p}^{*} y^{\prime}\right)=0
$$

because the top sequence above is exact, and

$$
i^{*}\left(j^{*} \bar{p}^{*} y^{\prime}\right)=p^{*} j^{\prime *} y^{\prime}=p^{*} h^{*}(\bar{y})=f^{*} \alpha^{*}(\bar{y})=y .
$$


Hence $j^{*} \bar{p}^{*} y^{\prime}$ is $H^{*}\left(B_{G} ; Q\right)$-free. But by the Borel Localization Theorem [14] we have

$$
\sum_{j=1}^{m} S^{-1} i_{j}^{*}: S^{-1} i^{*} H_{G}^{*}(M ; Q) \simeq S^{-1} H_{G}^{*}(F ; Q),
$$

where $S=H^{*}\left(B_{G} ; Q\right)--\{0\}$. Hence $S^{-1} \sum i^{*}\left[j^{*} \bar{p} y^{\prime}\right] \neq 0$, where $\left[j^{*} \bar{p} y^{\prime}\right]$ denotes the class of $j^{*} \bar{p} y^{\prime}$ in $S^{-1} H_{G}^{*}(M ; Q)$. But $S^{-1} \sum i_{j}^{*}\left[j^{*} \bar{p} y^{\prime}\right]=0$ by (1). This is a contradiction. Hence there exists at least one component $F_{0}$ of $F$ such that

$$
\left(f \mid F_{0}\right)^{*}: H^{k}(K(\pi, 1) ; Q) \rightarrow H^{k}\left(F_{0} ; Q\right)
$$

is non-trivial. Therefore $F_{0}$ is an aspherical cohomology $A_{k}(\pi)$-manifold. If $\pi$ is torsion free and $\chi(M) \neq 0$, a similar result is proved in [6].

Theorem 2.3. Let $M$ be an aspherical $A_{k}(\pi)$-manifold and $\pi^{\prime}$ is torsion free. Suppose that $\chi(M) \neq 0$, or $p(w, z) \neq 0$ for some $w$ and $z$. If $G$ is a compact Lie group acting effectively on $M$, then $G$ is finite group.

Proof. Suppose $G$ is not finite, then $G$ contains a toral subgroup $T^{s}, s \geq 1$, and $T^{s}$ still acts effectively on $M$. By hypothese, the action of $T^{s}$ on $M$ has nonempty fixed point set. Hence by Corollary $1.2, f_{*} H_{i}(M ; Q)=0$ for $i>n-r$. On the other hand, $f_{*}: H_{n}(M ; Q) \rightarrow H_{n}(K(\pi, 1) ; Q)$ is non-trivial. This is contradiction because $r \geq 1$.

As an easy corollary we have the following result. The case $\chi(M) \neq 0$ is the main theorem of Conner and Montgomery in [3].

Theorem 2.4. Let $G$ be a compact Lie group acting effectively on a closed connected aspherical manifold $M$ with $\chi(M) \neq 0$, or $p(w, z) \neq 0$ for some $w$ and $z$. Then $G$ is finite.

If we define the degree of symmetry $N_{T}(M)$ of $M$ as the supremum of the dimensions of all Lie groups which can act effectively on $M$. Then we have the following result from Theorem 2.3 and 2.4 .

Theorem 2.5. Let $M$ be an aspherical $A_{k}(\pi)$-manifold and $\pi^{\prime}$ is torsion 
free. Suppose that $\chi(M) \neq 0$. Then $N_{T}(M)=0$. In particular, if $M$ is an aspherical manifold with $\chi(M) \neq 0, N_{T}(M)=0$.

\section{Semi-Simple Degree of Symmetry}

Suppose that $M$ is a compact connected $n$-manifold. The semi-simple degree of symmetry $N_{T}^{s}(M)$ (resp. $N^{s}(M)$ ) of $M$ is defined as the supremum of the dimensions of all compact semi-simple Lie groups which can act effectively (resp. effectively and smoothly) on $M$. The semi-simple degree of symmetry has an interesting connection with the Riemannian geometry, that is, if $N^{s}(M) \neq 0$, then $M$ admits a Riemannian metric with positive scalar curvature [11].

Theorem 3.1. Let $M$ be an aspherical $A_{k}(\pi)$-manifold. If $\pi^{\prime}$ is torsionfree. Then $k \leq n-r$. Hence

$$
N_{T}^{s}(M) \leq(n-k)(n-k+1) / 2 .
$$

Proof. Let $G$ be a compact semi-simple Lie group acting effectively on $M$ with $\operatorname{dim} G=N_{t}^{s}(M)$. Since $\pi_{1}(G)$ is finite, so is $f_{*} i_{*} \pi_{1}(G)$. Hence $f_{*} H_{i}(M ; Q)=0$ for $i>n-r$. But $f_{*} H_{k}(M ; Q) \neq 0$, hence $r \leq n-k$. Let $G(x)$ be a principal orbit. Then $\operatorname{dim} G(x)=r \leq n-k$. But the group acts effectively on $G(x)$, hence

$$
\operatorname{dim} G \leq(n-k)(n-k+1) / 2 .
$$

This gives the desired conclusion.

Let $N=K(\pi, 1)$. If $N$ is a Riemannian $n$-manifold of negative curvature, and $M, f$ are both smooth, Theorem 3.1 in this special case was proved by Scheon and Yau in [13]. In [4], Donnelly and Schultz remove the smoothness condition. The general case with $\pi$ torsion-free was proved in [9]. A similar result for paracompact space $M$ and $\pi$ torsion-free was proved by Berstein in [1]. 
Theorem 3.2. Let $M$ be an asperical $A_{k}(\pi)$-manifold with $N_{T}^{s}(M)=0$. Suppose $\pi^{\prime}$ is torsion-free, $N_{T}(M) \neq 0$ and $\chi(M) \neq 0$. Then

$$
N_{T}(M) \leq \min (n-k,[n / 2])
$$

Proof. Since $N_{T}^{s}=0$, let $T^{s}$ act effectively on $M$, where $s=N_{T}(M)$. Since $\chi(M) \neq 0, F\left(T^{s}, M\right) \neq \emptyset$. Again, by using Corollary 1.2 we can verify that $k \leq n-r$. But for an effective toral group action, the principal isotopy subgroups are finite, hence $r=s$. It follows that $s \leq n-k$. Since $F \neq \emptyset$, from a result of $H$. T. Ku [6], we also have $s \leq[n / 2]$. This proves the theorem. A similar result was also proved in [9] under the following hypotheses: $M$ aspherical $A_{k}(\pi)$-manifold, $N_{T}^{s}(M)=0, N_{T}(M) \neq 0, \chi(M) \neq 0, \pi_{1}(M)$ abelian and $\pi$ torsion-free.

\section{References}

[1] I. Berstein, "On Covering space and Lie group actions", Contemporay Math., 37 (1985), 11-13.

[2] W. Browder and W. C. Hsiang, "G-actions and fundamental group", Invent. Math., 65 (1982), 411-424.

[3] P. E. Conner and D. Montgomery, "Transformation groups an $K(\pi, 1)$ ", I. Michigan Math., J. 6 (1059), 405-412.

[4] H. Donnelly and R. Schultz, "Compact group actions and maps in to aspherical manifolds", Topology, 21 (1982), 443-455.

[5] M. Gromov and H. B. Lawson, "Spin and scalar curvature in the presence of a fundamental group", I, Ann. of Math., (2) 111 (1980), 209-230.

[6] H. T. Ku, "Group actions on aspherical $A_{k}(\pi)$-manifolds with non-zero Euler characteristics", Proc. Amer. Math. Soc., 90 (1984), 459-462.

[7] H. T. Ku, "A generalization of the Conner inequalities", Proc. Conf. Transformations Groups, Springer-Verlag, Berlin and New York, (1968), 401-414.

[8] H. T. Ku and M. C. Ku, "Group actions on $A_{k}(\pi)$-manifolds", Trans. Amer. Math. Soc., 245 (1978), 469-492.

[9] H. T. Ku and M. C. Ku, "Group actions on aspherical $A_{k}(\pi)$-manifolds", Trans. Amer. Math. Soc., 278 (1983), 841-859.

[10] H. T. Ku and M. C. Ku, "The Pontrjagin numbers of an orbit map and generalised Gsignature theorem, transformations groups", Lecture Notes in Math., 1375 (1989), 198206.

[11] H. B. Lawson and S. T. Yau, "Scalar curvature, non-abelian group actions, and the degree of symmetry of exotic spheres", Comment. Math. Helv., 49 (1974), 232-244.

[12] D. Montgomery and Samelson, "Groups transitive on $n$-dimensional torus", Bull. Amer. Math. Soc., 49 (1943) 455-456. 
[13] R. Scheon and S. T. Yau, "Compact group actions and the topology of manifolds with non-positive curvature", Topology, 18 (1979), 361-380.

Department of Electrical and Computer Engineering, University of Massachusetts, Amherst, MA. 01003 , U. S. A. 